\title{
Dynamique non linéaire du marché mondial des huiles et des graisses \\ M Tancrède Voituriez
}

\section{Résumé}

L'étude de la dynamique des prix fournit des informations intéressantes sur les conséquences à attendre d'un changement des structures concurrentielles et d'une ouverture des marchés sur l'instabilité. Nous avons mené cette étude sur le marché mondial des huiles. Si l'hypothèse d'un pur chaos déterministe ne peut être acceptée, la présence d'une dynamique non linéaire très forte est démontrée. Elle confirme l'hypothèse d'une instabilité endogène, autogénérée, une instabilité propre au fonctionnement du marché.

\section{Abstract \\ Nonlinear dynamics in world vegetable oils prices}

Price dynamics analysis provides key information on the possible consequences one can expect from the opening up and the liberalization of agricultural markets. The potential role of chaotic dynamics in generating world vegetable oils prices is investigated here with more than 1400 weekly data. The evidence of a pure chaotic determinism cannot be accepted, while the proof of nonlinear dynamics in prices remains strong and casts some doubt on the market's ability to find out its equilibrium position and stabilize by itself.

\section{Citer ce document / Cite this document :}

Voituriez Tancrède. Dynamique non linéaire du marché mondial des huiles et des graisses. In: Économie rurale. №243, 1998. Modélisation des marchés agricoles. pp. 22-29;

doi : https://doi.org/10.3406/ecoru.1998.4989

https://www.persee.fr/doc/ecoru_0013-0559_1998_num_243_1_4989

Fichier pdf généré le 08/05/2018 
Les dernières discussions de l'OMC à Singapour et les orientations retenues par le Fair Act américain en 1996 accordent au marché une place fondamentale : dans son acception la plus simple d'un lieu de confrontation de l'offre et de la demande, il lui incombe de guider le producteur dans ses choix de culture en fournissant par l'intermédiaire des prix une indication claire de la rareté relative des produits. Implicitement, les cours mondiaux d'un produit agricole seront un indicateur de rareté d'autant plus précis qu'à travers le monde, le nombre des prix fixés s'amenuisera, puis s'annulera, et avec eux, les perturbations délétères qu'ils induisent. Tester l'hypothèse d'une stabilisation des cours à mesure que le marché se déleste des entraves au libre-échange et à la concurrence est délicat en raison de l'appréciation toujours incomplète du niveau de perfection concurrentielle qu'un tel marché a pu atteindre. Toutefois l'étude de la nature, endogène ou exogène, de la variabilité des prix fournit des informations intéressantes sur les conséquences à attendre d'un changement des structures concurrentielles sur l'instabilité. Nous avons mené cette étude sur le marché mondial des huiles.

Les mesures et les tests de non linéarité et de chaos ont été appliqués aux séries de prix hebdomadaires de six produits représentatifs: les huiles de palme, soja, tournesol, arachide, coco et une graisse, le suif, enregistrés à Sumatra puis en Malaisie (palme) et à Rotterdam du $1^{\mathrm{er}}$ janvier 1970 au 2 janvier 1997.

Les mesures ont été effectuées par l'estimation de l'exposant de Lyapunov et de la dimension de l'attracteur (procédure de Grassberger et Procaccia) sur les séries différenciées ; le test de bruit blanc de Brock, Dechert et Scheinkman (test BDS) a été appliqué ensuite aux résidus d'un modèle ARMA ajusté aux différences premières de chaque série et aux résidus normalisés d'un modèle ARMA-GARCH $(1,1)$. La présence d'une dynamique non linéaire très forte est démontrée. Elle confirme l'hypothèse d'une instabilité endogène, autogénérée, une instabilité propre au fonctionnement du marché.

La première partie de l'article est consacrée aux applications économiques du chaos. La seconde partie est employée à l'identification de la dynamique d'un marché particulier, le marché mondial des huiles et graisses. Enfin lieu nous avançons quelques hypothèses explicatives des principaux résultats.

\section{Applications du chaos à l'économie}

La première identification du chaos déterministe est habituellement attribuée au météorologue Lorenz (1963). Son modèle décrit une météo miniature par un système dynamique à trois variables, $\mathrm{X}, \mathrm{Y}, \mathrm{Z}$, et trois équations différentielles non linéaires parfaitement déterministes.

$\mathrm{dX} / \mathrm{dt}=10(\mathrm{Y}-\mathrm{X})$

$\mathrm{dY} / \mathrm{dt}=-\mathrm{XZ}+28 \mathrm{X}-\mathrm{Y}$

$\mathrm{dZ} / \mathrm{dt}=-\mathrm{XY}-8 / 3 \mathrm{Z}$

Les conditions initiales $\mathrm{X}_{0}, \mathrm{Y}_{0}, \mathrm{Z}_{0}$ et les trois équations décrivant l'évolution du système suffisent à déterminer son état en n'importe quel instant. Lorenz, fort de cette certitude, entreprit un jour de lancer une seconde fois ses simulations, non pas depuis l'origine $\left(\mathrm{X}_{0}, \mathrm{Y}_{0}, \mathrm{Z}_{0}\right)$, mais à partir d'un point intermédiaire de la série fournie par l'ordinateur. Les valeurs affichées sont d'abord conformes à celles produites lors du premier calcul, mais passé un certain délai, elles s'en écartent et se mettent à diverger. Pour quelle raison, puisque le système est complètement déterministe ? L'ordinateur n'écrit à l'écran que des valeurs arrondies. En entrant ses nouvelles coordonnées, Lorenz commet une erreur infime, du rang de l'arrondi, qui grossit exponentiellement et affecte l'état final du système. Ce phénomène d'instabilité locale est connu sous le nom de sensibilité aux conditions initiales. C'est une des deux propriétés fondamentales du chaos. La seconde tient dans le désordre produit. Ce désordre n'est qu'apparent : quoique imprévisible, la suite des états du système ne s'écarte pas d'une région de l'espace vers laquelle elle est attirée et converge. Cette région, ou attracteur, a la particularité de posséder une structure étrange, lacunaire - on dit encore «feuilletée »-, traduction des étirements (divergence locale) et repliements (convergence globale) que l'évolution du système lui impose.

L'hypothèse fort répandue de fluctuations produites par des chocs exogènes stochastiques, exprimée par la somme d'une « innovation " aléatoire gaussienne et d'une ten- 
dance linéaire, n'a jamais rencontré de vérification complètement satisfaisante sur les variables économiques que sont par exemple les PIB, les prix de certaines denrées agricoles ou les valeurs d'actifs' ${ }^{\prime}$. Le faible coefficient de corrélation lors de l'estimation des tendances, la distribution non gaussienne des fluctuations, invalidaient l'hypothèse en contrariant toute prévision. La théorie du chaos, en accommodant désordre et déterminisme, arrivait à point nommé pour prolonger l'espoir de l'économiste de comprendre le chahut des marchés, et l'espérance du financier d'accroître ses gains avec un risque mesuré. C'est la raison pour laquelle son application s'est d'abord observée dans les modèles de croissance et les milieux financiers ${ }^{2}$.

L'état d'un système est donné par un point dans un espace que l'on appelle espace des phases. L'ensemble des états successifs du système quand le temps varie forme la trajectoire, ou orbite. Le chaos déterministe étant défini par deux propriétés essentielles dans l'espace des phases (divergence locale, convergence globale), les grandeurs observées (PIB, taux de change, prix etc.) doivent traduire ou démentir ces propriétés. Comment procède-t-on ? On suppose dans un premier temps que la série de données étudiée constitue l'unique coordonnée observable d'un système dynamique de dimension inconnue. Comment déterminer ensuite si ce système est régulier, chaotique, ou très largement contaminé par du bruit et donc indissociable du hasard?

Grassberger et Procaccia (1983) ont proposé la solution suivante, en adoptant la méthode des retards de Takens (1980). Le signal de la coordonnée $X$ est observé à des intervalles de temps $\Delta t$, soit $X=\left(x_{0}, x_{1}, \ldots, x_{T}\right)$. De nouvelles variables sont construites à partir de $\mathrm{X}$, de longueur $n$ faible (en général 3,4 ou 5): $\mathrm{X}_{0}=\left(\mathrm{x}_{0}, \mathrm{x}_{1}, \ldots, \mathrm{x}_{\mathrm{n}-1}\right), \mathrm{X}_{1}$ $=\left(x_{1}, x_{2}, \ldots, x_{n}\right), X_{2}=\left(x_{2}, x_{3}, \ldots, x_{n+1}\right)$ etc. La trajectoire dans l'espace des phases prend les coordonnées $X_{1}, \ldots, X_{n}$. L'attracteur est reconstruit dans l'espace de dimension $n$ appelée dimension de "plongement » (embedding dimension) grâce aux recouvrements successifs des points $\mathrm{X}_{\mathrm{i}}$, tels qu'ils viennent d'être définis, par de sphères (ou hypersphères) de rayon $r$ de plus en plus faible. Le nombre de points $C_{n}(\mathrm{r})$ dans chaque sphère (ou hypersphère) varie comme $r^{v}$ où $v$ est la dimension de corrélation de l'objet étudié. En pratique, $s$ 'il existe une pente $v$ du rapport $\log \mathrm{C}_{n}(\mathrm{r}) / \log (\mathrm{r})$ indépendante de la dimension de plongement $n(n>v)$ à mesure que $n$ s'accroît, alors $v$ peut être considéré comme une mesure de la dimension de l'attracteur:

$$
v=\lim _{r \rightarrow 0} \ln \left(C_{n}(r)\right) / \ln (r)
$$

On l'appelle dimension de « corrélation ». Elle vaut 2.05 dans le modèle de Lorenz et plus généralement est qualifiée de fractale toutes les fois qu'elle prend une valeur non

\footnotetext{
1. On se reportera pour mémoire aux échanges entre Nelson et Plosser (1982). Cochrane (1988) et Perron (1989).

2. Pour un récapitulatif récent sur cycles et chaos en économie, voir par
} exemple Nishima et Sorger (1996), Benhabib (1996). entière. La reconstruction de l'attracteur et l'estimation de sa dimension permettent d'éprouver la convergence globale d'un système.

La divergence locale, l'autre propriété fondamentale du chaos, est décelée à travers l'estimation du plus grand exposant de Lyapunov. Les exposants de Lyapunov indiquent le taux moyen de séparation des orbites correspondants à des points initiaux proches les uns des autres. L'algorithme d'estimation de ces exposants sur une série réelle univariée est dû à Wolf et al. (1985). On n'en rapporte ici que le principe. Deux points : $X_{i}$ l'origine d'une orbite, et $\mathrm{X}_{\mathrm{j}}$ un point proche de $\mathrm{X}_{\mathrm{i}}$ par lequel l'orbite passe à un instant donné, sont choisis dans l'espace des phases. On compare la suite de l'orbite, après son passage en $\mathrm{X}_{\mathrm{j}}$, avec son début.

On calcule pour cela par une méthode des moindres carrés la matrice $\mathrm{M}_{\mathrm{i}}$ décrivant la transformation des vecteurs $\mathrm{X}_{\mathrm{i}}$ $X_{j}$ en vecteurs $X_{i+1} \cdot X_{j+1}$. Les exposants de Lyapunov $\lambda_{i}$ sont les valeurs propres du terme :

$\operatorname{Lim}_{n \rightarrow \infty}\left(M^{*}{ }_{i+n} \ldots M_{i}^{*} M_{i} M_{i+1} \ldots M_{i+n}\right)^{1 / n}$

où $\mathrm{M}^{*}$ désigne la matrice adjointe de $\mathrm{M}$ (Eckmann et al., 1985). La présence de $\lambda_{i}>0$ est la signature du chaos déterministe. En théorie, trois cas de figure sont possibles :

1. Le plus grand exposant de Lyapunov est positif et la dimension de l'attracteur est fractale : la dynamique du système est chaotique, par conséquent nécessairement non linéaire et déterministe.

2. A l'inverse, l'insensibilité aux conditions initiales (plus grand exposant de Lyapunov négatif ou nul : pas de divergence locale) et la convergence vers les figures connues de la dynamique linéaire que sont le point, le cycle limite et le tore seront la preuve qu'un système déterministe simple (linéaire) dirige la série ${ }^{3}$.

3. Enfin la divergence globale (dimension infinie de l'attracteur) est la manifestation d'un bruit prépondérant et d'un système aléatoire.

Un quatrième cas de figure existe en réalité. Les algorithmes récents ne permettent pas de mesurer convenablement la dimension d'un attracteur de dimension élevée (supérieure en pratique à 5 ou 6) à partir d'un échantillon réduit de données (moins de 2000 comme ici). Il est impossible alors, avec les deux mesures que sont la dimension de corrélation et l'exposant de Lyapunov, de trancher en faveur du chaos déterministe (cas 1) plutôt que d'un signal stochastique (cas 3). C'est cruellement ce cas de figure qui surgit le plus souvent dans les études de systèmes ouverts, comme les marchés économiques, contaminés par du bruit.

3. Dans un système linéaire, une instabilité locale est inobservable puisqu'elle est également globale : elle dégénère en effondrement ou en explosion à l’infini. 
Mais la difficulté de distinguer le hasard du chaos est progressivement surmontée par la construction de tests statistiques. Ces tests sont susceptibles d'extirper d'un signal perturbé par du bruit ou décrit comme un bruit blanc, un ordre sous-jacent. Le plus célèbre est le test BDS, écrit par Brock (1986), Brock et al. (1987, 1988, 1991) à partir de l'algorithme de Grassberger et Procaccia.

L'hypothèse nulle du test BDS est celle d'un bruit blanc. La statistique $\mathrm{W}$ de ce test converge asymptotiquement vers une loi normale centrée réduite $N(0,1)$ :

$\mathrm{W}=\mathrm{T}^{1 / 2}\left[\mathrm{C}_{n}-\left(\mathrm{C}_{1}\right)^{n}\right] / \mathrm{b}_{\mathrm{n}}$

Où $\mathrm{C}_{n}$ est défini tel qu'en [4], $\mathrm{T}$ la longueur de l'échantillon et $b_{n}$ est la variance asymptotique de $T^{1 / 2}\left[C_{n}-\right.$ $\left(C_{1}\right)^{n}$ ] (se reporter à Brock (1991)). Ce test permet de nuancer, voire d'invalider, les résultats des tests classiques de bruit blanc qui ne s'intéressent qu'aux corrélations de premier ordre. Précisément, il permet d'apprécier la qualité d'une modélisation non linéaire de type $A R C H$ ou GARCH en testant la blancheur des résidus après correction de leur hétéroscédasticité.

\section{Application au marché des huiles et des graisses}

A. Dimension de corrélation : L'estimation de la mesure de l'attracteur selon l'algorithme de Grassberger et Procaccia (dimension de corrélation) est effectuée sur chacune des séries différenciées des prix hebdomadaires suivants, du 1-1-1970 au 2-1-1997 (soit 1410 données par série) : prix de l'huile de palme fob port Malais, de l'huile de soja fob ex-mill en Hollande, de l'huile de tournesol fob port N. Ouest Europe, de l'huile d'arachide toute origine caf Rotterdam, de l'huile de coco Philippines caf Rotterdam et enfin du suif USA caf Rotterdam, fournis par Oil World.

La dimension de l'espace de plongement ( $n$ dans [4] supra) varie de 1 à 10 . Une condition de fiabilité de la mesure $v$ de l'attracteur dans ces espaces successifs est que $n$ soit supérieur ou égal à $2 v+1$. Les attracteurs de dimension supérieure à 5 ne sont donc pas considérés ici, ce principe de précaution n'exprimant rien de plus que la difficulté de dissocier hasard et chaos dans des systèmes à nombre élevé de degrés de liberté.

Résultats : L'absence d'une limite supérieure, d'une asymptote horizontale, de la dimension estimée lorsque la dimension de plongement $n$ s'accroît ne permet pas de conclure à la présence d'un attracteur; elle se situe entre 5 et 6 , qui est la zone critique. Rien ne permet donc de distinguer chaque série d'une série engendrée par le hasard. Le graphique 1 , avec en abscisse la dimension de plongement (Embedding dimension) et en ordonnée l'estimation de la dimension de corrélation grimpant le long de la première diagonale illustre ce résultat (détail des mesures en annexe 1).
B. Exposant de Lyapunov: On choisit un espace de plongement de dimension $n=10$. Le rayon $r$ de l'hypersphère est fixé à $10^{-4}$, la longueur des trajectoires à trois points. Le tableau 1 fournit la valeur estimée $\lambda^{*}$ du plus grand exposant de Lyapunov mesuré en bit/unité de temps à partir des six séries brutes. L'écart entre deux trajectoires proches augmente de $\lambda^{*}$ entre deux unités de temps (respectivement diminue si $\lambda^{*}$ est négatif). La valeur maximale est l'unité (doublement de l'écart). Rappelons qu'en théorie une valeur positive signale le chaos.

\section{Tableau 1. Estimation du plus grand exposant}

de Lyapunov $\lambda *$

Prix hebdomadaires différenciés, 1970 : 1:1 - $1997: 2: 1$

\begin{tabular}{ccccccc}
\hline & Palme & Soja & Tourn. & Arach. & Coco & Suif \\
\hline $\mathrm{I}^{\star}$ & .048 & .049 & .036 & .053 & .037 & .073 \\
& $(.015)$ & $(.014)$ & $(.015)$ & $(.016)$ & $(.016)$ & $(.016)$ \\
\hline
\end{tabular}

Résultats : $\lambda *>0$ : la dynamique des prix est chaotique. Potentiellement. L'absence de test statistique sur $\lambda *$ ne permet pas pour l'instant de rejeter l'hypothèse selon laquelle la divergence observée serait uniquement l'effet d'un bruit prépondérant dans le signal. Cette hypothèse eût été rejetée en cas de stabilité globale, qu'hélas nous n'observons pas. Le test BDS est susceptible de dissiper cette incertitude.

\section{HASARD OU CHAOS : TESTS DE BRUIT BLANC}

Chaque série est débarrassée de ses composantes identifiables par les outils traditionnels des séries temporelles : extraction d'une tendance linéaire (modèles ARMA) puis standardisation des résidus en cas d'hćtćroscédasticité (modèle non linéaire GARCH) ${ }^{1}$. Les résidus de la série filtrée sous ARMA, les résidus de la série filtrée sous ARMA-GARCH, sont passés au crible du test BDS. Rappelons l'hypothèse nulle de ce test : la série est un bruit blanc.

a) Ecriture du modèle : l'hypothèse d'une racine unitaire et d'une tendance stochastique ne pouvant être rejetée, un modèle linéaire classique $\mathrm{AR}(\mathrm{p})$ est construit à partir de chaque série différenciée $X_{t}$ :

cte $+B(L) X_{t}=e_{t}$,

où $B(L)=b_{1} L+\ldots+b_{p} L^{p} ; L$ est l'opérateur de retard tel que $L^{s} X_{t}=X_{t-s} ; e_{t}$ est distribué selon une loi normale de moyenne nulle et de variance $h_{t}$.

L'ordre $p$ du modèle AR est évalué grâce au critère d'Akaike. L'équation [7] est ensuite estimée selon la méthode du maximum de vraisemblance, en considérant d'abord que la variance $h_{\mathbf{t}}$ des résidus est indépendante du temps : $h_{t}=h$ (homoscédasticité). Puis on suppose que la variance dépend du temps: $h_{t}=a_{0}+a_{1} e 2_{t-1}+a_{2} h_{t-1}$ (hétéroscédasticité) ${ }^{2}$.

1. Pour une synthèse sur l'analyse des produits agricoles au moyen de séries temporelles, se reporter par exemple à Myers (1994) et Tavéra (1991).

2. Voir Bollerslev (1986). 
Les résultats de l'estimation du modèle [7] sont présentés dans l'annexe 2. La validité de l'estimation est confirmée par les tests de corrélation de premier ordre sur les résidus. Ainsi du test de Ljung et Box, qui ne permet pas de rejeter l'hypothèse nulle d'un bruit blanc.

Tableau 2. Test de bruit blanc - statistique $Q^{\star}$ de Ljung-Box ${ }^{1}$ $\Delta \log$ (Prix hebdomadaires, $1970: 1: 1$ - $1997: 2: 1$ )

\begin{tabular}{lcc}
\hline Série - Modèle & $Q^{*}$ & Probabilité critique \\
\hline Palme - AR (7) & 279.88 & .9946 \\
\hline Soja - AR (43) & 270.11 & .8754 \\
\hline Tournesol - AR (24) & 268.53 & .9864 \\
\hline Arachide - AR (16) & 301.62 & .8831 \\
\hline Coco - AR (27) & 293.35 & .8357 \\
\hline Suif - AR (1) & 380.04 & .1374 \\
\hline
\end{tabular}

${ }^{1}$ Le test est rejeté avec $1 \%, 5 \%$ et $10 \%$ d'erreur lorsque la probabilité critique est inférieure respectivement à.01,.05 et.10.

L'hétéroscédasticité (corrélation du second ordre) des résidus est ensuite testée. On applique pour cela la procédure ARCH proposée par Engle (1982) sur chaque résidu $e_{t}$.

\section{Tableau 3. Test ARCH (1) sur résidus1}

\begin{tabular}{lcccccc}
\hline Modèle & Palme & Soja & Tourn & Arach & Coco & Suif \\
\hline AR $(p)$ & $189.86^{* * *}$ & $38.35^{* * *}$ & $17.52^{* * *}$ & $4.90^{* *}$ & $16.26^{\star * *}$ & $35.14^{\star * *}$ \\
\hline
\end{tabular}

${ }^{1}$ L'hypothèse nulle est celle d'absence d'autocorrélation des variances. Les statistiques du test sont distribuées selon un $\chi 2$ (1).

$* \star *=$ significatif à $1 \%$

** $=$ significatif à $5 \%$

L'hypothèse d'hétéroscédasticité ne peut être rejetée. Les six séries de résidus comportent toutes une autocorrélation du second ordre : leur variance $h_{t}$ change avec le temps. La variabilité d'aujourd'hui est corrélée à la variabilité d'hier, conclusion qui rejoint des observations similaires de Fulponi (1994) sur différents produits agricoles dont le soja. Un modèle non linéaire AR (p)-GARCH $(1,1)$ est construit pour tenir compte de cette particularité. La persistance d'hétéroscédasticité dans le cas du suif après correction par GARCH $(1,1)$ est levée grâce à un modèle de rang $2, \operatorname{AR}(\mathrm{p})-\mathrm{GARCH}(2,2)$. Les estimations sont rassemblées dans l'annexe 2 .

On dispose à présent des trois séries : la série brute différenciée, la série $e_{t}$ des résidus $A R(p)$, et la série $u_{t}$ des résidus standardisés AR-GARCH ${ }^{1}$, auxquelles nous allons appliquer le test BDS de bruit blanc.

\section{b) Test BDS}

La dimension de plongement varie de 1 à 10 , le rayon de l'hypersphère est fixé à 1 fois l'écart type de la série étudiée. Sous l'hypothèse nulle d'un bruit blanc, la statistique $\mathrm{W}$ converge asymptotiquement vers la loi $\mathrm{N}(0,1)$. Les valeurs critiques du test à $5 \%$ et $1 \%$ sont donc respectivement 1.96 et 2.33 (tableau 4). Dans le cas de rési-

1. Les résidus standardisés $u_{t}$ sont donnés par : $u_{t}=e_{t} h_{t}^{-1 / 2}$, où $h_{t}$ est la variance conditionnelle. dus GARCH, la distribution empirique du test diffère très légèrement d'une loi $\mathrm{N}(0,1)$; les valeurs critiques sont tirées de Brock et al. (1991) (tableau 5).

Tableau 4. Test BDS sur résidus AR (p)

\begin{tabular}{lrrrrrr}
\hline$n$ & PALME & SOJA & TOURN & ARACH & COCO & SUIF \\
2 & $12.68^{* *}$ & $9.67^{* *}$ & $6.27^{* *}$ & $11.10^{* * *}$ & $8.43^{* *}$ & $9.48^{* \cdots}$ \\
3 & $13.96^{* *}$ & $11.00^{* *}$ & $8.03^{* *}$ & $13.05^{* * *}$ & $9.76^{* *}$ & $12.27^{* *}$ \\
4 & $16.01^{* *}$ & $12.05^{* *}$ & $9.49^{* *}$ & $15.17^{* * *}$ & $10.69^{* *}$ & $14.56^{* *}$ \\
5 & $18.05^{* * *}$ & $13.75^{* * *}$ & $10.56^{* *}$ & $17.12^{* * *}$ & $12.22^{* *}$ & $16.05^{* *}$ \\
10 & $31.47^{* *}$ & $24.34^{* *}$ & $15.30^{* *}$ & $32.01^{* *}$ & $19.35^{* *}$ & $32.13^{* *}$ \\
\hline$* * *$ significatif à $1 \%$ & & & &
\end{tabular}

L'hypothèse de bruit blanc est fortement rejetée sur les résidus $\mathrm{AR}$. On teste à présent les résidus standardisés d'un modèle AR-GARCH afin de déterminer si la correction des corrélations du second ordre blanchit le bruit.

\section{Tableau 5. Test BDS sur résidus AR (p)-GARCH $(1,1)+$}

\begin{tabular}{|c|c|c|c|c|c|c|}
\hline$n$ & PALME & SOJA & TOURN & ARACH & COCO & SUIF \\
\hline 2 & $1.88^{\star \star}$ & $2.34^{\star * *}$ & 1.18 & $-2.27^{\star * \star}$ & $1.56^{\star}$ & $2.08^{* * *}$ \\
\hline 3 & .887 & $2.78^{* * *}$ & $1.36^{*}$ & $-2.74^{\star * *}$ & $1.48^{* *}$ & $4.59^{* \star *}$ \\
\hline 4 & $1.05^{*}$ & $2.74^{* * *}$ & $1.58^{* *}$ & $-2.71^{* \star *}$ & $1.25^{\star}$ & $5.99^{* * *}$ \\
\hline 5 & $1.77^{\star \star \star}$ & $3.20^{* * *}$ & $1.77^{\star \star \star}$ & $-2.48^{* \star \star}$ & $1.36^{* *}$ & $6.98^{* * *}$ \\
\hline 10 & $2.32^{* \star *}$ & $7.95^{\star \star \star}$ & $2.06^{\star \star}$ & $-2.51^{\star * *}$ & 1.22 & $12.97^{\star \star *}$ \\
\hline
\end{tabular}

L'hypothèse de bruit blanc est à nouveau rejetée dans l'ensemble, hormis dans le cas du coco, dont le modèle non linéaire AR (27)-GARCH $(1,1)$ paraît représenter convenablement la dynamique des prix. Les résultats du test sur les cinq autres séries de résidus confirment la présence d'une composante non linéaire très forte, potentiellement chaotique.

En résumé : De chaque série de prix différenciée, considérée comme l'unique variable observable d'un système dynamique, ont été mesurées deux grandeurs clés : la stabilité globale, l'instabilité locale, afin de déceler l'existence d'une structure déterministe simple (faible degré de liberté). Alors que la stabilité globale n'a pas été prouvée (dimension de l'attracteur située dans la zone critique, entre 5 et 6), l'instabilité locale est démontrée (exposant de Lyapunov positif). Ce premier résultat rejette clairement l'hypothèse d'un pur chaos déterministe et avec elle, l'hypothèse d'une faculté du marché à se stabiliser de luimême.

Le test BDS de bruit blanc sur les résidus de type ARGARCH montre qu'une structure non linéaire forte subsiste dans les résidus de cinq séries parmi six : l'instabilité des prix est endogène et susceptible, par amplification non linéaire, de déboucher sur des booms et des crises sans que n'intervienne aucun choc extérieur.

\section{Hypothèses explicatives}

Un système non linéaire comporte des équilibres multiples; la sensibilité aux conditions initiales rend illusoire la reproduction d'une série réelle à partir d'une simulation ; enfin il ne tolère pas la prévision. Trois bon- 
nes raisons de conserver la vision d'un monde parfaitement linéaire, perturbé ici et là par le vacarme de quelques pétards sous une cloche gaussienne. En plus de créer des problèmes énormes d'estimation mathématique, les non linéarités soulèvent des difficultés de compréhension : d'où viennent-elles ? On peut avancer quelques explications.

1. Accrochages en fréquence. Basiquement, des productions oléagineuses de périodes différentes coexistent dans les deux hémisphères : pluriannuelles, annuelles et pérennes, tandis que des pics saisonniers de consommation (ramadan, nouvel an chinois, hiver européen) ponctuent le calendrier de la demande. Au total, l'offre et la demande physiques s'agrègent dans le temps.

2. Accrochages en fréquence (suite). L'agrégation de l'offre et de la demande est renforcée par la variabilité de l'horizon des décisions d'achat et de vente des agents sur le marché. Cet horizon est multiple : une heure pour le spéculateur, jusqu'à plus d'un an pour certains industriels. Lorsque les anticipations se rejoignent, des accrochages en fréquence des achats et des ventes se produisent, amplifiant les variations de prix.

3. Des anticipations nécessaires. Le retrait récent de l'Etat de la gestion des grandes plantations (palme) et surtout des importations (ouverture progressive à la concurrence des importations indiennes et chinoises par exemple) replace les anticipations au coeur de l'échange. Là où par exemple la State Trading Corporation indienne importait en fonction seulement des déficits courants de la production oléagineuse locale, des importateurs privés raisonneront en terme de coûts d'opportunité et d'anticipations de l'état futur du marché. Ce point renforce le précédent.

4. Rigidité de la demande. La demande globale d'huiles et graisses est rigide tandis qu'au sein de chaque industrie les substitutions entre huiles s'effectuent à l'intérieur de seuils ou de «butoirs» pour reprendre le terme de Hicks. Et l'on sait que l'existence de seuils est mathématiquement propice à l'apparition de dynamiques non linéaires.

L'étude de phénomènes oscillatoires en physique a montré que le couplage de deux oscillateurs non linéaires quasi-périodiques de fréquence différente peut, à travers l'apparition imprévisible de crêtes ou d'accrochages en fréquence, dégénérer en dynamique non linéaire ou chaotique'. On sait d'autre part que la contrainte de seuils de certains paramètres d'un modèle et la contrainte de non négativité des stocks d'un agent privé sont propices à l'apparition d'une dynamique non linéaire ${ }^{2}$. Enfin Bous-

1. Se reporter par exemple à Bergé et Dubois (1992).

2. Sur ce dernier point, voir Deaton et Laroque (1990). sard (1996) a montré que la confrontation d'une demande globale rigide et d'une offre averse au risque pouvait produire et entretenir des chroniques chaotiques sur un marché agricole. Les quatre points précédents pourraient ainsi convenablement expliquer la dynamique du marché. Leur formulation mathématique au sein d'un même modèle appelle de nouveaux travaux.

\section{Conclusion}

Les résultats présentés démontrent la non linéarité de la dynamique des prix des huiles de palme, soja, tournesol, coco, arachide et du suif. Ils infirment dans tous les cas la présence d'un pur chaos déterministe. L'étude du chaos et de la dynamique non linéaire des prix en économie rurale a derrière elle de notables antécédents qui appellent de nouvelles recherches et ont motivé celle-ci : citons l'exhibition de chaos à partir de cobweb par Boussard (1996) ; dans les marchés à terme de produits agricoles (sucre et café) par DeCoster et al. (1992), dans les prix du boeuf aux USA, par Kohzadi et al. (1995) et les travaux de Chavas (1991) sur le cycle du porc aux USA. La première conclusion de ce travail n'est guère originale: de nouveaux tests statistiques doivent être construits afin de distinguer le hasard du chaos dans les systèmes de dimension limite (entre 5 et 6 degrés de libertés) et remplacer efficacement l'estimation de la dimension de l'attracteur, dénoncée par Granger comme une statistique visuelle. Les résultats permettent toutefois de répondre à certaines questions :

- il n'existe ni cycle régulier, ni tendance dans la dynamique des prix observés ;

- la sensibilité aux conditions initiales ruine tout espoir de prévision ;

- le marché est fondamentalement instable. Booms et crises font partie de sa nature, si ce terme a un sens en économie ;

- l'effet est disproportionné à la cause : un même écart entre l'offre et la demande ne produit jamais une même variation de prix.

Enfin, l'amplitude variable des prix rend le coût d'accès au marché comparable à une barrière d'entrée, sans qu'il soit nécessaire d'invoquer externalités ou rendements croissants. L'histoire du marché des huiles depuis la fin du $\mathrm{XIX}^{\mathrm{e}}$ montre du reste qu'il n'existe pas d'exemple d'oléagineux apparus dans le commerce mondial sans coup de force préalable de l'État ${ }^{3}$.

Tancrède VOITURIEZ • CIRAD Montpellier

3. Voituriez (1997), à paraître. 


\section{RÉFÉRENCES BIBLIOGRAPHIQUES}

Akaike H. Information Theory and an Extension of the Maximum Likelihood Principle. Second International Symposium on Information Theory, ed. B.N. Petrov and F. Csàki, Budapest, Academiai Kiado, 1973.

Benhabib J. On Cycles and Chaos in Economics. In Studies in Nonlinear Dynamics and Econometrics, 1996, 1 (1), pp. 1-2.

Bergé $\mathrm{P}$. et Dubois M. Chaos déterministe expérimental et attracteurs étranges. In Dalmedico A.D., Chabert J.-L. et K. Chemla (dir.), 1992, Chaos et Déterminisme, Seuil, Paris, pp. 115-169.

Bollerslev T. Generalized Autoregressive Conditional Heteroscedasticity. In Journal Econometrics, 1986, 31, pp. 307-327.

Boussard J.-M. When Risk Generates Chaos. In Journal of Economic Behaviour and Organization, 1996, 29, pp. 433-446.

Brock W.A. Distinguishing Random and Deterministic Systems : Abridged Version. In Journal Econometrics Theory, 1986, 40, pp. 168-95.

Brock W.A., Dechert W.D. et Scheinkman J.A. A Test of Independence Based on the Correlation Dimension. Working Paper 8702, Social Systems Research Institute, University of Wisconsin-Madison, 1987.

Brock W.A. Sayers C.L. Is the Business Cycle Characterized by Deterministic Chaos? In Journal Monetary Economics, 1988, 22, pp. 71-90.

Brock W.A., Hsieh D.A. et Le Baron B. Dynamics, Chaos, and Instability : Statistical Theory and Economic Evidence. MIT Press, 1991.

Chavas J.-P. et Holt M.T. On Nonlinear Dynamics: The Case of the Pork Cycle. In American Journal of Agricultural Economics, août 1991, pp. 819-828.

Deaton A. et Laroque G. On the Behavior of Commodity Prices. NBER Working Paper, septembre 1990, \# 3439.

DeCoster G.P., Labys W.C. et Mitchell D.W. Evidence of Chaos in Commodity Futures Prices. In The Journal of Futures Markets, 1992, vol. 12, n³, pp. 291-305.

Dickey D. et Fuller W.A. Distribution of the Estimators for Time Series Regressions with a Unit Root. In Journal of the Americain Statistical Association, 1979, 47, pp. 427 431.

Engle R.Autoregressive Conditional Heteroscedasticity with Estimates of the Variance of United Kingdom Inflation. In Econometrica, 1982, 55, pp. 391-407.
Fulponi L. La variabilité des prix internationaux de base : les marchés sont-ils efficaces? In Économie Rurale, 1994, 219, pp. 16-23.

Grassberger P. et Procaccia I. Characterization of Strange Attractors. In Physical Revue Letters, 1983, 50, pp. 448451.

Grassberger P. An optimized box-assisted algorithm for fractal dimensions. In Physics Letters A, 1990, vol. 148, $\mathrm{n}^{\circ} 1-2$, pp. 63-68.

Kazuo N. et Sorger G. Optimal Cycles and Chaos : A Survey. In Studies in Nonlinear Dynamics and Econometrics, 1996, 1 (1), pp. 11-28.

Kiel L. D et Elliott E. (éd.). Chaos Theory in the Social Sciences. The University of Michigan Press, 1996.

Kohzadi N. et Boyd M.S. Testing for Chaos and Nonlinear Dynamics in Cattle Prices., In Canadian Journal of Agricultural Economics, 1995, 43, pp. 475-484.

Ljung G.M. et Box G.E.P. On a Measure of Lack of Fit in Time Series Models. In Biometrika, 1978, 67, pp. 297 303.

Lorenz E.N. Deterministic nonperiodic flow. In Journal of the Atmospheric Sciences, 1963, 20, pp. 130-142.

Myers R.J. Time Series Econometrics and Commodity Price Analysis : A Review. In Review of Marketing and Agricultural Economics, 1994, vol. 62, n² 2, pp. 167-181. Nelson Ch. et Plosser Ch. Trend and Random walks in Macro-economics Time Series. In Journal of Monetary Economics, 1982, vol. 10.

Perron P. The Great Crash, the Oil Price Shock and the Unit Root Hypothesis. In Econometrica, novembre 1989.

Takens F. Detecting Strange Attractors in Turbulence. In Dynamic Systems and Turbulence, ed. D. Rand and L. Young. Lecture Notes in Mathematics, 1980, Berlin : Springer-Verlag, $n^{\circ} 898$.

Tavéra C. Tests de racine unité et stationnarisation des séries non stationnaires : présentation générale et application au cas des séries agricoles. In Économie et Prévision, 1991, vol. 3, n 99, pp. 67-80.

Tong H. A personal Overview of Nonlinear Time Series Analysis from a Chaos Perspective. In Scandinavian Journal of Statistics, 1995, vol. 22, pp. 399-445.

Voituriez T. La vie du gras. A paraître.

Wolf A., Swift J.-B., Swinney H.L. et Vastano J.A. Determining Lyapunov Exponents from a Time Series. In Physica, 1985, 16D, pp. 285-317. 
ANNEXE 1. Dimension de l'attracteur (dimension de "Corrélation ")

Série nominale différenciée des prix hebdomadaires. 1970:1:1 - 1997:2:1

\begin{tabular}{lcccccrrrr}
\hline & $n=2$ & $n=3$ & $n=4$ & $n=5$ & $n=6$ & $n=7$ & $n=8$ & $n=9$ & $n=10$ \\
\hline Palme & 2.38 & 3.01 & 3.82 & 4.11 & 4.70 & 5.02 & 5.47 & 5.84 & 5.84 \\
& $(.97)$ & $(.21)$ & $(.26)$ & $(.27)$ & $(.27)$ & $(5.02)$ & $(5.47)$ & $(5.84)$ & $(.34)$ \\
\hline Soja & 1.94 & 2.97 & 3.75 & 4.27 & 4.92 & 5.32 & 5.75 & 6.29 & 6.22 \\
& $(.53)$ & $(.07)$ & $(.14)$ & $(.11)$ & $(.12)$ & $(5.32)$ & $(5.75)$ & $(6.29)$ & $(.45)$ \\
\hline Tournesol & 1.68 & 2.94 & 3.82 & 4.35 & 5.01 & 5.38 & 5.90 & 6.07 & 6.46 \\
& $(.89)$ & $(.15)$ & $(.11)$ & $(.08)$ & $(.12)$ & $(5.38)$ & $(5.90)$ & $(.31)$ & $(.33)$ \\
\hline Arachide & .97 & 2.64 & 3.62 & 3.97 & 4.10 & 4.45 & 4.99 & 5.27 & 5.14 \\
& $(.61)$ & $(.84)$ & $(.50)$ & $(.08)$ & $(.27)$ & $(.28)$ & $(.447)$ & $(.38)$ & $(5.14)$ \\
\hline Coco & 1.85 & 2.91 & 3.71 & 4.36 & 4.80 & 4.94 & 5.29 & 5.61 & 5.51 \\
& $(.84)$ & $(.09)$ & $(.18)$ & $(.18)$ & $(4.8)$ & $(.27)$ & $(.334)$ & $(.36)$ & $(5.51)$ \\
\hline Suif & .79 & 2.08 & 3.53 & 4.21 & 4.74 & 5.22 & 5.67 & 5.54 & 5.85 \\
& $(.24)$ & $(.41)$ & $(.21)$ & $(.24)$ & $(.32)$ & $(.50)$ & $(.57)$ & $(5.54)$ & $(5.85)$ \\
\hline
\end{tabular}

La valeur de l'écart type est entre parenthèses ; $n$ est la dimension de plongement ou « embedding " dimension.

Source des prix : Oil World Publication.

ANNEXE 2. Estimations des modèles AR et AR -GARCH ${ }^{1,2}$
Alog (Prix hebdomadaires, 1970:1:1-1997:2:1)

Palme

\begin{tabular}{lll}
\hline & AR (7) & AR $(7)$-GARCH $(1,1)$ \\
\hline b1 & .3819 & $.0900^{\star \star \star}$ \\
b4 & $.0812^{\star \star \star}$ & $.0749^{\star \star}$ \\
b6 & -.0001 & $-.0628^{\star \star}$ \\
b7 & $-.0659 \star \star$ & $-.0636^{\star \star}$ \\
GARCH, $a_{0}$ & & $6.768 \mathrm{e}^{\star 0}-05^{\star \star \star}$ \\
GARCH, $\mathrm{a}_{1}$ & & $.7759^{\star \star \star}$ \\
GARCH, $\mathrm{a}_{2}$ & & $.2224^{\star \star \star}$ \\
Convergence & 1 & 1 \\
\hline
\end{tabular}

Soja

\begin{tabular}{lll}
\hline & AR (43) & AR (43)-GARCH $(1,1)$ \\
\hline$b_{1}$ & $.0640^{\star \star}$ & .0318 \\
$b_{5}$ & $-.0641^{\star \star}$ & $-.0685^{\star \star}$ \\
$b_{7}$ & $-.0781^{\star \star \star}$ & .0191 \\
$b_{10}$ & -.0219 & $-.0535^{\star}$ \\
$b_{16}$ & $-.045^{\star}$ & -.0434 \\
$b_{17}$ & .0418 & $.0679^{\star \star}$ \\
$b_{21}$ & -.0254 & $-.0442^{\star}$ \\
$b_{22}$ & $.0610^{\star \star}$ & $.0643^{\star \star}$ \\
$b_{31}$ & $-.0501^{\star}$ & -.0331 \\
$b_{32}$ & $.0545^{\star \star}$ & .0173 \\
$b_{35}$ & .0389 & $.0483^{\star}$ \\
$b_{38}$ & .0271 & $.0462^{\star}$ \\
$b_{43}$ & $.0815^{\star \star \star}$ & $.0614^{\star \star \star}$ \\
GARCH, $a_{0}$ & & $2.997 e^{-0} 5^{\star \star \star}$ \\
GARCH, & & $.8804_{1}^{\star \star \star}$ \\
GARCH, a & & $.1426^{\star \star \star}$ \\
Convergence & 1 & 1
\end{tabular}

Tournesol

\begin{tabular}{lll}
\hline & AR $(24)$ & AR $(24)-$ GARCH $(1,1)$ \\
\hline$b_{1}$ & $.1954^{\star \star \star}$ & $.1495^{\star \star}$ \\
$b_{3}$ & $.0501^{\star}$ & $.0563^{\star}$ \\
$b_{6}$ & $.0587^{\star \star}$ & .0259 \\
$b_{7}$ & $-.0531^{\star}$ & -.0385 \\
$b_{20}$ & -.0265 & $-.0494^{\star \star}$ \\
$b_{24}$ & $.0848^{\star \star \star}$ & $.0810^{\star \star \star}$ \\
GARCH, a & & $3.772 e^{\star *}-05^{\star \star \star}$ \\
GARCH, a & & $.8811^{\star \star \star}$ \\
GARCH, a & & $.11677^{\star \star \star}$ \\
Convergence & 1 & 1 \\
\hline
\end{tabular}

Arachide

\begin{tabular}{|c|c|c|}
\hline & AR (16) & AR $(16)$-GARCH $(1,1)$ \\
\hline $\begin{array}{l}b_{1} \\
b_{3} \\
b_{6} \\
b_{7} \\
b_{8} \\
b_{10} \\
b_{12} \\
b_{13} \\
b_{15} \\
b_{16} \\
\text { GARCH, a } \\
\text { GARCH, a } \\
\text { GARCH, a2 } \\
\text { Convergence }\end{array}$ & $\begin{array}{l}.2858^{\star \star \star} \\
-.1581^{\star \star \star} \\
.1298^{\star \star \star} \\
-.0697^{\star \star} \\
.0269 \\
.0710^{\star \star} \\
-0535^{\star} \\
.0621^{\star \star} \\
.0441 \\
-0539^{\star \star}\end{array}$ & $\begin{array}{l}.2560^{\star \star \star} \\
.0341 \\
-.0355 \\
-.0414^{\star} \\
.0839^{\star \star \star} \\
.0684^{\star \star \star} \\
.0533^{\star \star \star} \\
.0359 \\
.0640^{\star \star \star \star} \\
-.0441^{\star \star} \\
9.188 \mathrm{e}^{\circ}-05^{\star \star \star} \\
.5303^{\star * \star} \\
.4052^{\star \star \star} \\
1\end{array}$ \\
\hline
\end{tabular}

Coco

\begin{tabular}{|c|c|c|}
\hline & AR (27) & AR (27)-GARCH $(1,1)$ \\
\hline $\begin{array}{l}b_{1} \\
b_{8} \\
b_{12} \\
b_{14} \\
b_{18} \\
b_{23} \\
b_{24} \\
b_{27} \\
\text { GARCH, a } \\
\text { GARCH, a } \\
\text { GARCH, a }\end{array}$ & 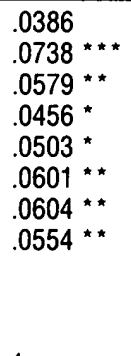 & $\begin{array}{l}.0630^{\star \star} \\
.0177 \\
.0495^{\star} \\
.0333 \\
.0175 \\
.0097 \\
.0508^{\star \star} \\
.0249 \\
9.83 e^{-05^{\star \star \star}} \\
.8219^{\star \star \star \star} \\
.1794^{\star \star \star}\end{array}$ \\
\hline Convergence & 1 & 1 \\
\hline
\end{tabular}

Suif

\begin{tabular}{lll}
\hline & AR (1) & AR $(1)-$ GARCH $(1,1)$ \\
\hline $\mathrm{b}_{1}$ & $.0937^{\star \star \star}$ & $.1543^{\star \star \star}$ \\
GARCH, a & & $8.392 \mathrm{e}^{\mathrm{e}-06^{\star \star \star}}$ \\
GARCH, $\mathrm{a}_{1}$ & & $.8888^{\star \star \star}$ \\
GARCH, a & & $.1139^{\star \star \star}$ \\
Convergence & 1 & 1 \\
\hline
\end{tabular}

1. Le terme constant, toujours non significatif, n'a pas été retenu lors des estimations.

2. La variance conditionnelle s'écrit : $h_{t}=a_{0}+a_{1} e 2_{t-1}+a_{2} h_{t-1}$, (e le résidu du modèle AR) 
Palme

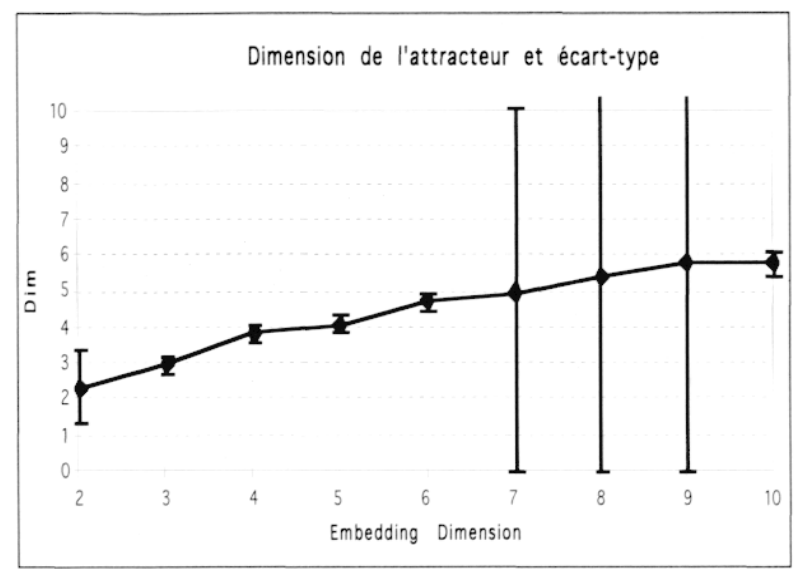

Soja

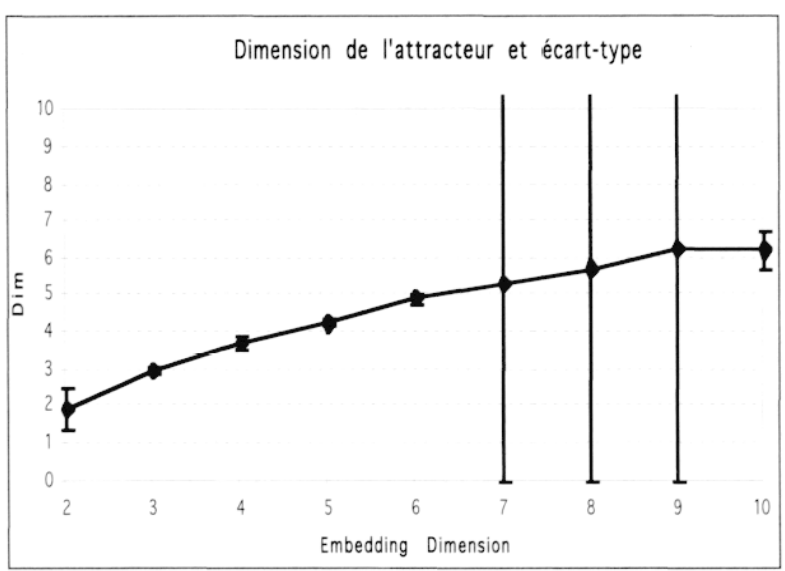

Tournesol

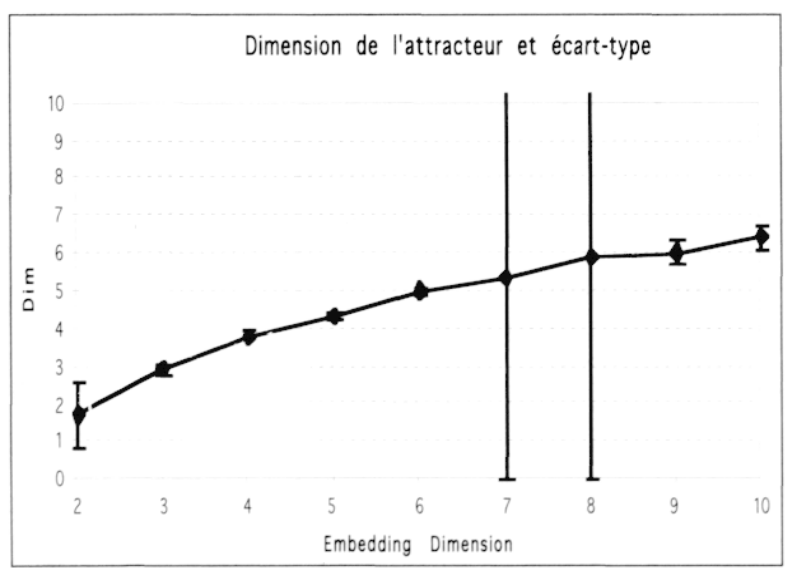

\section{Arachide}

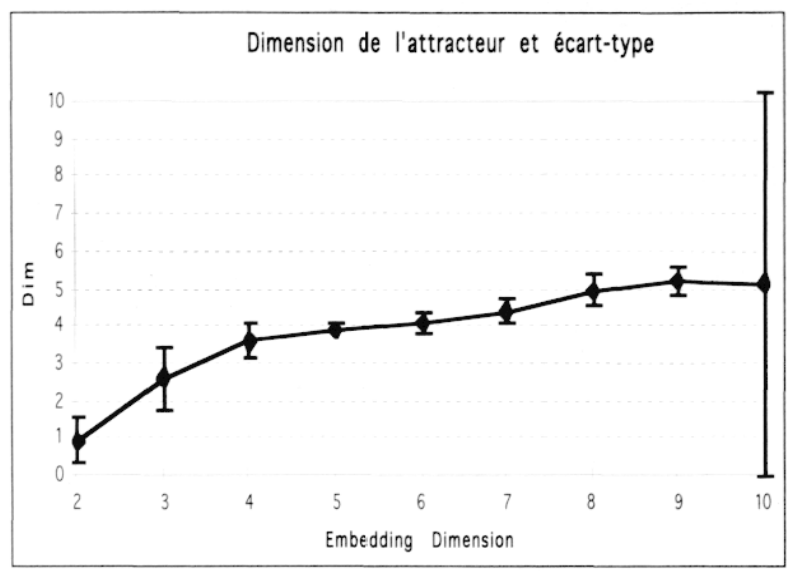

Coco

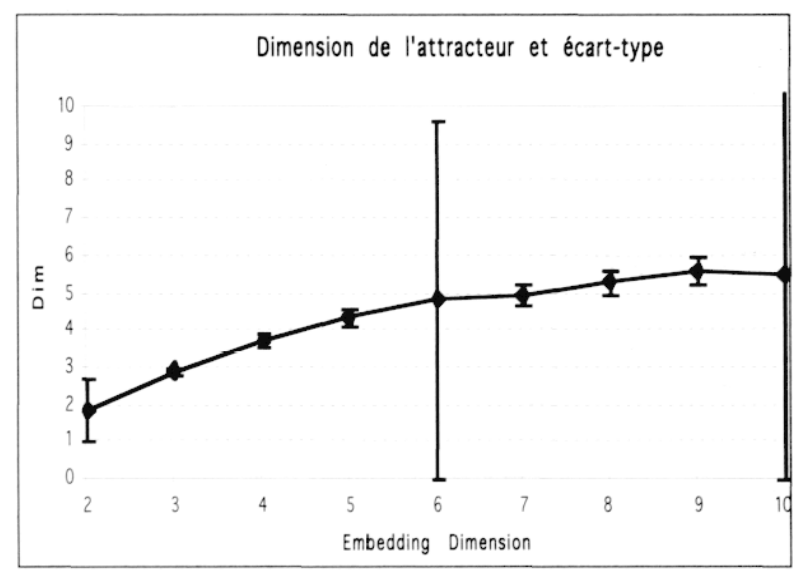

Suif

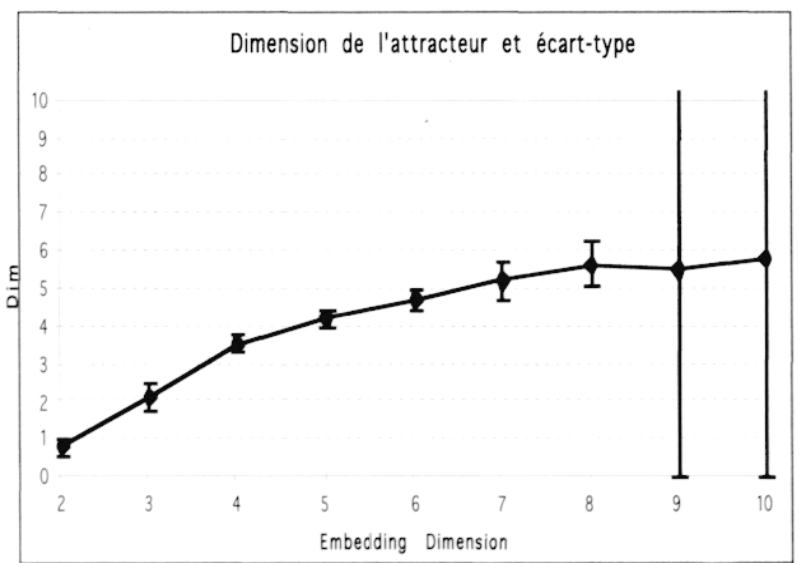

